\title{
SPECTRAL PROPERTIES OF THIN-FILM PHOTONIC CRYSTALS*
}

\author{
ALEX FIGOTIN ${ }^{\dagger}$ AND YURI A. GODIN ${ }^{\dagger}$
}

\begin{abstract}
We study spectral properties of three-dimensional photonic crystals formed by a periodic array of air cubes separated by a thin film of optically dense dielectric material. The thickness $\delta$ of the dielectric component is assumed to be small, whereas its permittivity $\varepsilon$ is large. The spectrum of the photonic crystal is studied as $\delta \rightarrow 0$ while $\varepsilon \delta=\eta^{-1}$ is kept constant. Under the additional condition of a vanishing normal component of the magnetic field on the surface of the optically dense dielectric film, we carry out a thorough analytical and numerical analysis of the spectrum of the photonic crystal. In particular, for small $\eta$ we obtain the linear and quadratic approximations of the spectral bands and, given $\delta$, we estimate the minimum value of $\varepsilon$ for which at least one spectral gap opens up.
\end{abstract}

Key words. photonic crystal, electromagnetic waves, spectral bands and gaps, thin dielectric films, Maxwell's equations, periodic structure

AMS subject classifications. 35B27, 65F15, 73D25, 78A45

\section{PII. S0036139900372831}

1. Introduction. Photonic crystals - man-made periodic dielectric structureshave received a great deal of attention for the last decade because of their capabilities to manipulate, in a number of ways, the propagation of electromagnetic waves. There is a steadily growing number of publications on theoretical and experimental studies of one-, two-, and three-dimensional photonic crystals. In particular, the studies include band-gap properties of the spectra of photonic crystals, localized and defect states arising from perturbation of photonic crystals, and novel ways to control the propagation of electromagnetic ways using fundamental properties of photonic crystals.

The theory of photonic crystals is a significant part of their design and fabrication. It is based on the classical Maxwell equations for spatially inhomogeneous dielectric media, i.e., the media with the permittivity $\varepsilon(\boldsymbol{r})$ and permeability $\mu(\boldsymbol{r})$ dependent on the position vector $\boldsymbol{r}$. For perfect photonic crystals, functions $\varepsilon(\boldsymbol{r})$ and $\mu(\boldsymbol{r})$ are periodic, and, hence, the Floquet-Bloch theory can be applied. The theory yields general spectral properties such as the Bloch form for the eigenmodes and band structure of the spectrum. Naturally, the next step is to ask how relevant physical quantities depend on the geometry of the photonic crystal and, say, the values of permittivity $\varepsilon$ of its constitutive components. For instance, if a photonic crystal consists of two components of permittivities $\varepsilon$ and 1, a practical question one can ask is, What is the minimum value $\varepsilon_{\min }$ such that for appropriately chosen crystal geometry at least one gap opens up? Alternatively, if $\varepsilon>\varepsilon_{\min }$ is given, what geometry provides the largest gap, if any? Unfortunately, for two- and three-dimensional structures these natural questions are hard to answer both theoretically and numerically. The reason

\footnotetext{
*Received by the editors May 8, 2000; accepted for publication (in revised form) December 12, 2000; published electronically April 18, 2001. This research was sponsored by the Air Force Office of Scientific Research, Air Force Materials Command, under grant F49620-99-1-0203. The U.S. Government is authorized to reproduce and distribute reprints for governmental purposes notwithstanding any copyright notation thereon. The views and conclusions contained herein are those of the authors and should not be interpreted as necessarily representing the official policies or endorsements, either expressed or implied, of the Air Force Office of Scientific Research or the U.S. Government.

http://www.siam.org/journals/siap/61-6/37283.html

$\dagger$ Department of Mathematics, University of California, Irvine, CA 92697-3875 (afigotin@uci.edu, ygodin@math.uci.edu).
} 
of the theoretical difficulties stems from the fact that there are no exactly solvable nontrivial models of periodic dielectric structures in the dimensions two or three for which one can describe analytically the spectral properties based on the Maxwell equations. Numerical computation of the band-gap structure, on the other hand, requires considerable computer resources. Spectral computations are complicated by the medium discontinuity, since real photonic crystals are usually made of two materials with different permittivity or permeability. In addition to that, the stronger the discontinuity, the more pronounced are the effects of multiple scattering, such as emergence of spectral gaps and localization, if defects are introduced.

Taking into account the above problems, the theory, we believe, can be developed at least in two directions. The first direction is related to the study of some extreme (asymptotic) situations [1], [12], [14], [15], [16], [24], [36], which, on one hand, can provide an explanation of physical phenomena in photonic crystals and, on the other hand, can be treated analytically. That is a common approach in theoretical physics, and the "only" problem is finding those extreme situations which satisfy the above criteria. The second direction is to develop mathematical approaches which would provide a solid ground for constructions of time-efficient robust numerical algorithms which explicitly take into account the medium discontinuities and the divergence-free condition of the related fields. The primary subject of this paper is related to the first direction.

The three-dimensional case is the most difficult in every respect, including experiments, fabrication, theoretical analysis, and computations. In spite of all challenges, there has been a considerable amount of publications in recent years covering a wide range of activities: from new ideas in fabrication of the crystals and new ways to employ their properties in optical devices [3], [4], [5], [6], [7], [9], [10], [17], [18], [19], [21], [22], [25], [27], [31], [37], [40], [41], [42], [43], [44], [46] to theoretical and computational studies allowing accurate computation of the properties of photonic crystals [8], [30], [28], [29], [38], [39], [45].

In this paper we study the spectral properties of three-dimensional photonic crystals formed by a periodic array of air cubes separated by a thin film of optically dense dielectric material. We consider the asymptotic situation when the thickness $\delta$ of the dielectric component approaches zero and its permittivity $\varepsilon$ approaches infinity so that $\varepsilon \delta=\eta^{-1}$ is kept constant. Under the additional condition of a vanishing normal component of the magnetic field at the surface of the optically dense dielectric film, we carry out a thorough analytical and numerical analysis of the spectrum of the photonic crystal.

Thin films or screens made up of a dielectric substances of large permittivity (real or imaginary) similar to ones described above have been considered in electromagnetic waves scattering problems [4], [5]. In those papers as well as in the paper [33] on electromagnetic theory of gratings, operator methods were proposed involving, in particular, impedance and admittance operators and Calderon projectors. These operators were efficient for numerical and asymptotic analysis. Among other relevant asymptotic models allowing substantial theoretical approach, mention should be made of structures built from thin wires [26], [32] or thin lossy stripes [34].

1.1. Statement of the results. In this paper we conduct a thorough study of photonic crystals formed by a periodic array of cubic air cavities, separated by thin films of optically dense lossless dielectric (see Figure 1.1). It has been shown [13] (see also [11]) that if (i) the dielectric film of permittivity $\varepsilon$ allows only magnetic fields tangential to its surface and (ii) its thickness $\delta$ is chosen appropriately depending on $\varepsilon$, 


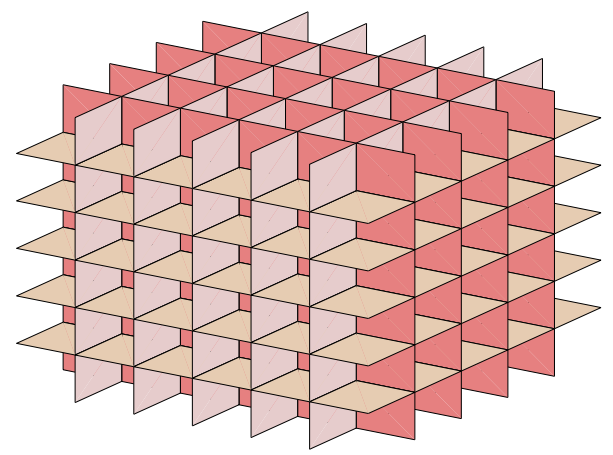

FIG. 1.1. A slab of three-dimensional photonic crystal composed of cubic air cavities separated by thin dielectric plates of high dielectric constant.

then the spectrum of the photonic crystal has narrow bands and, consequently, large spectral gaps. Here we give more elementary proofs of all results needed from [13] that make this paper completely self-contained. Assuming smallness of the parameter $\eta=1 / \varepsilon \delta$ we carry out a detailed investigation of the asymptotic model. In particular, based on the analytic perturbation theory we compute the first and the second order terms of the asymptotic approximations of the spectrum branches. We also carry out numerical computations of the spectral bands. Comparison of the numerical results with the analytic perturbation theory of the second order shows that they are in good agreement up to the point when the spectral gaps close down. Hence, we conclude, spectral gaps can be adequately analyzed by the classical perturbation theory up to the point of their closure.

The paper is organized as follows. In section 2 we describe basic properties of eigenmodes and eigenfrequencies of a single cubic cavity (resonator) with a vanishing normal magnetic field component on the boundary. Then we show that the photonic crystal in question can be viewed as a periodic array of weakly coupled resonators with the coupling constant $\eta=1 / \varepsilon \delta$. Assuming that the coupling constant $\eta$ is small, we show in section 3 that (i) the spectral bands of such photonic crystal are proportional to $\eta$ and (ii) the spectral bands are situated about the eigenvalues of the cubic resonator. Consequently, appreciable spectral gaps will arise for sufficiently small $\eta$. In section 4 we reduce the spectral analysis of the photonic crystal to the spectral analysis of an infinite matrix $\mathscr{M}_{0}+\eta \mathscr{M}_{1}$, where $\mathscr{M}_{0}$ is a diagonal matrix corresponding to the periodic array of completely decoupled cubic cavities, while the perturbation matrix $\mathscr{M}_{1}$ is a sparse matrix describing the energy of coupling. Then in section 5 we prove that matrix $\mathscr{M}_{1}$ is relatively compact with respect to $\mathscr{M}_{0}$ by showing that $\mathscr{M}_{0}^{\frac{1}{2}} \mathscr{M}_{1} \mathscr{M}_{0}^{\frac{1}{2}}$ is the sum of three compact operators related to the directions along $x_{1}, x_{2}$, and $x_{3}$ axes. This decomposition allows us to get constructive truncation error estimates. The final sections are devoted to the computation of the spectrum. In particular, we use the analytic perturbation theory to find the exact linear bounds for the asymptotic behavior of the eigenvalues as $\eta \rightarrow 0$. It turns out that if an eigenvalue corresponds to two eigenmodes of different polarizations, then the linear upper bound of this eigenfrequency as $\eta \rightarrow 0$ is exactly $\xi+16 \eta$, where $\xi$ is the corresponding eigenvalues of the single resonant cavity. Otherwise, if all eigenmodes corresponding to the unperturbed eigenvalue $\xi$ have one polarization, then the upper linear bound of the perturbed eigenvalue is exactly $\xi+12 \eta$. Numerical computation of 
the spectral bands shows that all gaps are closing when $\eta=1 / \varepsilon \delta \approx 2$ or, equivalently, $\varepsilon \delta \approx 1 / 2$. We have found that the quadratic approximation of the bands

$$
2 \pi^{2} \leq \xi_{1} \leq 2 \pi^{2}+12 \eta-2.01 \eta^{2}, \quad 3 \pi^{2} \leq \xi_{2} \leq 3 \pi^{2}+16 \eta-2.31 \eta^{2}, \ldots
$$

is in good agreement with the numerical results. Based on these computations, we readily derive the following simple formula for the minimum value of the permittivity $\varepsilon_{\min }$ for which at least one gap opens up:

$$
\varepsilon_{\min }=\frac{1}{2\left[1-(1-f)^{1 / 3}\right]},
$$

where $f$ is the filling fraction of the optically dense dielectric.

2. Spectral properties of a cubic cavity. Consider first electromagnetic oscillations in a unit cubic cavity $Q_{0}: 0<x_{i}<1, \quad i=1,2,3$, with perfectly conducting walls. It is convenient to represent the electric induction $\boldsymbol{D}(\boldsymbol{x}, t)$ and the magnetic field $\boldsymbol{H}(\boldsymbol{x}, t)$ in the cavity through the divergent-free vector potential $\boldsymbol{\Phi}(\boldsymbol{x}, t)$ such that

$$
\boldsymbol{D}(\boldsymbol{x}, t)=\nabla \times \boldsymbol{\Phi}(\boldsymbol{x}, t), \quad \boldsymbol{H}(\boldsymbol{x}, t)=\frac{1}{c} \frac{\partial}{\partial t} \boldsymbol{\Phi}(\boldsymbol{x}, t),
$$

and

$$
\nabla \cdot \boldsymbol{\Phi}(\boldsymbol{x}, t)=0
$$

where $c$ is the speed of light. For the time-harmonic field

$$
\boldsymbol{\Phi}(\boldsymbol{x}, t)=e^{-i \omega t} \mathbf{\Psi}(\boldsymbol{x})
$$

equations (2.1)-(2.3) turn into

$$
\begin{aligned}
& \Delta \boldsymbol{\Psi}(\boldsymbol{x})+\xi \boldsymbol{\Psi}(\boldsymbol{x})=0, \quad \xi=\frac{\omega^{2}}{c^{2}}, \\
& \nabla \cdot \boldsymbol{\Psi}(\boldsymbol{x})=0,\left.\quad \boldsymbol{n} \cdot \boldsymbol{\Psi}(\boldsymbol{x})\right|_{\Gamma_{0}}=0
\end{aligned}
$$

where $\Gamma_{0}$ is the surface of the cube and $\boldsymbol{n}$ is the vector normal to the surface $\Gamma_{0}$. The eigenvalues of the cubic resonator satisfying (2.4)-(2.5) are given by

$$
\xi=\pi^{2}\left(p_{1}^{2}+p_{2}^{2}+p_{3}^{2}\right),
$$

while the eigenmodes $\boldsymbol{\Psi}_{\boldsymbol{p}}(\boldsymbol{x})$ are conveniently expressed by

$$
\boldsymbol{\Psi}_{\boldsymbol{p}}(\boldsymbol{x})=\boldsymbol{A}_{\boldsymbol{p}} \star \boldsymbol{\Theta}_{\boldsymbol{p}}(\boldsymbol{x})=\left(\begin{array}{c}
A_{p, 1} \sin \left(\pi p_{1} x_{1}\right) \cos \left(\pi p_{2} x_{2}\right) \cos \left(\pi p_{3} x_{3}\right) \\
A_{p, 2} \cos \left(\pi p_{1} x_{1}\right) \sin \left(\pi p_{2} x_{2}\right) \cos \left(\pi p_{3} x_{3}\right) \\
A_{p, 3} \cos \left(\pi p_{1} x_{1}\right) \cos \left(\pi p_{2} x_{2}\right) \sin \left(\pi p_{3} x_{3}\right)
\end{array}\right),
$$

where $\boldsymbol{A}_{\boldsymbol{p}}=\left(A_{p, 1}, A_{p, 2}, A_{p, 3}\right)$ is a real polarization vector corresponding to an integervalued nonzero index-vector $\boldsymbol{p}=\left(p_{1}, p_{2}, p_{3}\right)$,

$$
\boldsymbol{\Theta}_{\boldsymbol{p}}(\boldsymbol{x})=\left(\begin{array}{c}
\sin \left(\pi p_{1} x_{1}\right) \cos \left(\pi p_{2} x_{2}\right) \cos \left(\pi p_{3} x_{3}\right) \\
\cos \left(\pi p_{1} x_{1}\right) \sin \left(\pi p_{2} x_{2}\right) \cos \left(\pi p_{3} x_{3}\right) \\
\cos \left(\pi p_{1} x_{1}\right) \cos \left(\pi p_{2} x_{2}\right) \sin \left(\pi p_{3} x_{3}\right)
\end{array}\right),
$$


and $\boldsymbol{a} \star \boldsymbol{b}$ denotes three-dimensional vector

$$
\boldsymbol{a} \star \boldsymbol{b}=\left(\begin{array}{c}
a_{1} b_{1} \\
a_{2} b_{2} \\
a_{3} b_{3}
\end{array}\right) .
$$

Hereafter we suppose the eigenmodes $\boldsymbol{\Psi}_{p}(\boldsymbol{x})$ (2.7) to be normalized by the condition

$$
\int_{Q_{0}}\left\|\Psi_{p}(\boldsymbol{x})\right\|^{2} d \boldsymbol{x}=1
$$

That implies that if all $p_{1}, p_{2}, p_{3}$ are positive, then

$$
A_{p, 1}^{2}+A_{p, 2}^{2}+A_{p, 3}^{2}=8 .
$$

If one of $p_{j}, j=1,2,3$, equals zero, for instance, $p_{1}=0$, then normalized amplitudes obey

$$
A_{p, 2}^{2}+A_{p, 3}^{2}=4
$$

and we set the amplitude of the missing component of vector $\boldsymbol{\Psi}_{p}(\boldsymbol{x})$ to be zero $\left(A_{p, 1}=\right.$ 0 in the example). In addition to that, the divergence-free condition (2.5) implies

$$
\boldsymbol{A}_{\boldsymbol{p}} \cdot \boldsymbol{p}=0
$$

Due to this restriction, the eigenmode (2.7) has only two independent constants $A_{p, j}$, $j=1,2,3$, at most one of which can be zero. If none of the components of the index $\boldsymbol{p}=\left(p_{1}, p_{2}, p_{3}\right)$ equals zero, then the corresponding eigenvalue has multiplicity two. Alternatively, if one of the numbers $p_{1}, p_{2}$, or $p_{3}$ is zero, then the eigenvalue corresponding to such $\boldsymbol{p}$ is simple. Thus, the set of all indices $\mathcal{P}$ can be partitioned into two sets

$$
\mathcal{P}=\mathcal{P}_{ \pm} \cup \mathcal{P}_{0},
$$

where $\mathcal{P}_{ \pm}$contains only all-positive indices of multiplicity two, while $\mathcal{P}_{0}$ has its elements simple indices with one zero component. In what follows we will distinguish the indices $\left(p_{1}, p_{2}, p_{3}\right)$ corresponding to identical eigenvalues $\boldsymbol{p}^{2}=p_{1}^{2}+p_{2}^{2}+p_{3}^{2}$. First 11 eigenvalues (2.6) are given by

$$
2 \pi^{2}, 3 \pi^{2}, 5 \pi^{2}, 6 \pi^{2}, 8 \pi^{2}, 9 \pi^{2}, 10 \pi^{2}, 11 \pi^{2}, 12 \pi^{2}, 13 \pi^{2}, 14 \pi^{2} .
$$

In the above sequence there are missing integers because not every integer number is representable by a sum of two or three squares. Necessary and sufficient conditions for such representation are given in [20]. Notice that the eigenvalues (2.6) of the unit cubic cavity are the extreme values of the quadratic form

$$
\int_{Q_{0}}|\nabla \times \mathbf{\Psi}(\boldsymbol{x})|^{2} d \boldsymbol{x}=\left(M_{0} \boldsymbol{\Psi}, \boldsymbol{\Psi}\right), \quad \nabla \cdot \boldsymbol{\Psi}(\boldsymbol{x})=0,\left.\quad \boldsymbol{n} \cdot \boldsymbol{\Psi}(\boldsymbol{x})\right|_{\Gamma_{0}}=0,
$$

which defines a self-adjoint operator $M_{0}$ in the Hilbert space $L^{2}\left(Q_{0}\right)$. 
3. Asymptotic model of the photonic crystal. Let us denote by $Q_{m}$ the periodic array of cubes obtained by translation of the unit cube $Q_{0}$ by an integervalued vector $\boldsymbol{m}$ (see Figure 1.1):

$$
Q_{\boldsymbol{m}}=Q_{0}+\boldsymbol{m}, \quad \boldsymbol{m} \in \mathbb{Z}^{3} .
$$

Let also $\Gamma$ be the union of the cubes' faces having high dielectric constant $\varepsilon$. Then the vector potential $\Psi(\boldsymbol{x})$ defined by (2.1)-(2.3) satisfies the equation

$$
\nabla \times \frac{1}{\varepsilon(\boldsymbol{x})} \nabla \times \mathbf{\Psi}(\boldsymbol{x})=\xi \Psi(\boldsymbol{x}), \quad \xi=\frac{\omega^{2}}{c^{2}} .
$$

The eigenvalue problem (3.2) has a variational formulation in terms of the stationary values of the quadratic form

$$
\mathcal{M}(\eta)=\mathcal{M}_{0}+\eta \mathcal{M}_{1}
$$

where

$$
\begin{aligned}
& \left(\mathcal{M}_{0} \boldsymbol{\Psi}, \boldsymbol{\Psi}\right)=\int_{\mathbb{R}^{3}-\Gamma}|\nabla \times \boldsymbol{\Psi}(\boldsymbol{x})|^{2} d \boldsymbol{x}, \\
& \left(\mathcal{M}_{1} \boldsymbol{\Psi}, \boldsymbol{\Psi}\right)=\int_{\Gamma}\left|\boldsymbol{n}_{+} \times \boldsymbol{\Psi}_{+}(\boldsymbol{x})+\boldsymbol{n}_{-} \times \boldsymbol{\Psi}_{-}(\boldsymbol{x})\right|^{2} d S,
\end{aligned}
$$

and $\boldsymbol{\Psi}(\boldsymbol{x})$ satisfies the conditions

$$
\begin{aligned}
\nabla \cdot \boldsymbol{\Psi}(\boldsymbol{x}) & =0, \quad \boldsymbol{x} \in \mathbb{R}^{3}-\Gamma ; \\
\boldsymbol{n}_{ \pm} \cdot \boldsymbol{\Psi}_{ \pm}(\boldsymbol{x}) & =0, \quad \boldsymbol{x} \in \Gamma,
\end{aligned}
$$

where $\boldsymbol{n}_{+}=-\boldsymbol{n}_{-}$and $\boldsymbol{\Psi}_{ \pm}$are, respectively, the two opposite normal vectors to the surface $\Gamma$ and the field adjacent to $\Gamma$ areas. The model (3.3) was introduced in [13] (for more discussions see [11]). In the standard fashion we reduce the problem in the infinite domain to a finite cell $K$ using the Floquet-Bloch transform

$$
\boldsymbol{\Psi}(\boldsymbol{k}, \boldsymbol{x})=\sum_{\boldsymbol{m} \in \mathbb{Z}^{3}} \boldsymbol{\Psi}(\boldsymbol{x}+\boldsymbol{m}) e^{-i \boldsymbol{k} \cdot \boldsymbol{m}}, \quad \boldsymbol{x} \in Q_{0}, \quad \boldsymbol{k} \in K,
$$

where the quasi momentum $\boldsymbol{k}$ belongs to the Brillouin zone $K=[-\pi, \pi)^{3}$. This transform decomposes operator $\mathcal{M}$ into the fibers $M(\boldsymbol{k}, \eta)$ [35], [23]

$$
(\mathcal{M} \Psi)(\boldsymbol{k}, \eta)=M(\boldsymbol{k}, \eta) \boldsymbol{\Psi}(\boldsymbol{k}, \eta),
$$

where each fiber $M(\boldsymbol{k}, \eta)$ is a self-adjoint operator in the Hilbert space $L^{2}\left(Q_{0}\right)$

$$
M(\boldsymbol{k}, \eta)=M_{0}+\eta M_{1}(\boldsymbol{k}) .
$$

Operator $M_{0}$, given by (2.16) and (2.5), corresponds to a single cubic cavity with perfectly conducting walls. The perturbation operator $M_{1}(\boldsymbol{k})$ is defined by the quadratic form

$$
\left(M_{1}(\boldsymbol{k}) \boldsymbol{\Psi}, \boldsymbol{\Psi}\right)=\sum_{j=1}^{3} \int_{\gamma_{j}}\left|\boldsymbol{e}_{j} \times\left[\boldsymbol{\Psi}(\boldsymbol{x})-e^{i k_{j}} \mathbf{\Psi}\left(\boldsymbol{x}+\boldsymbol{e}_{j}\right)\right]\right|^{2} d S,
$$


where $\gamma_{j}$ are the faces of the unit cube $Q_{0}$ that are determined by the intersection $\gamma_{j}=\Gamma_{0} \bigcap\left\{x_{j}=0\right\}, j=1,2,3$, and $\boldsymbol{e}_{j}$ are the vectors of the standard basis.

According the Floquet-Bloch theory, the spectrum $\sigma(\mathcal{M})$ of the operator $\mathcal{M}$ is the union of spectra $\sigma(M(\boldsymbol{k}, \eta))$ as $\boldsymbol{k}$ runs the Brillouin zone $K=[-\pi, \pi)^{3}$. To calculate efficiently the spectrum $\sigma(M(\boldsymbol{k}, \eta))$ we observe that the operator $M_{0}$ has a compact resolvent and that $M_{1}(\boldsymbol{k})$ is compact relatively to $M_{0}$ [13], i.e., the operator

$$
\mathfrak{M}(\boldsymbol{k})=M_{0}^{-1 / 2} M_{1}(\boldsymbol{k}) M_{0}^{-1 / 2}
$$

is compact. This allows us to treat $\eta M_{1}(\boldsymbol{k})$ in (3.10) as a small perturbation of $M_{0}$ for $\eta \ll 1$. In section 5 we give an elementary alternative proof of the relative compactness based on the matrix representation of $\mathfrak{M}(\boldsymbol{k})$ that makes all the proofs in this paper completely self-contained.

From the properties of $M_{1}$ it follows that

(i) operator $M(\boldsymbol{k}, \eta)$ has a compact resolvent, and the spectrum $\sigma(M(\boldsymbol{k}, \eta))$ is discrete;

(ii) spectrum $\sigma(M(\boldsymbol{k}, \eta))$ approaches $\sigma\left(M_{0}\right)$ (2.6) uniformly in $\boldsymbol{k}$ as $\eta \rightarrow 0$; in particular, for small $\eta$ there must be gaps in the spectrum of the original operator $\mathcal{M}$ [13], [11];

(iii) spectral bands of $M(\boldsymbol{k}, \eta)$ lie above the corresponding point spectrum (2.6) of unperturbed operator $M_{0}$ since $M_{0} \leq M(\boldsymbol{k}, \eta)$.

3.1. Boundary values of the eigenmodes. Unlike operator $M_{0}$, the perturbation operator $M_{1}$ (3.11) is defined through the values of $\boldsymbol{\Psi}(\boldsymbol{x})$ on the boundary of the cubic cavity where expressions of the eigenmodes can be simplified. Since the normal component of the eigenmodes vanishes on the cube's surface we introduce vectors $\boldsymbol{\Theta}_{\boldsymbol{p}}^{(j)}(\boldsymbol{x})$ of tangential components of the eigenmodes $\boldsymbol{\Psi}_{\boldsymbol{p}}(\boldsymbol{x})(2.7)$ on the faces $x_{j}=0$ or $x_{j}=1, j=1,2,3$.

Case 1. All components of $\boldsymbol{p}=\left(p_{1}, p_{2}, p_{3}\right)$ are positive, i.e., $\boldsymbol{p} \in \mathcal{P}_{ \pm}$. Then we denote

$$
\begin{aligned}
& \boldsymbol{\Theta}_{\boldsymbol{p}}^{(1)}(\boldsymbol{x})=\left(\begin{array}{c}
0 \\
\sin \left(\pi p_{2} x_{2}\right) \cos \left(\pi p_{3} x_{3}\right) \\
\cos \left(\pi p_{2} x_{2}\right) \sin \left(\pi p_{3} x_{3}\right)
\end{array}\right), \boldsymbol{\Theta}_{\boldsymbol{p}}^{(2)}(\boldsymbol{x})=\left(\begin{array}{c}
\sin \left(\pi p_{1} x_{1}\right) \cos \left(\pi p_{3} x_{3}\right) \\
0 \\
\cos \left(\pi p_{1} x_{1}\right) \sin \left(\pi p_{3} x_{3}\right)
\end{array}\right), \\
& \boldsymbol{\Theta}_{\boldsymbol{p}}^{(3)}(\boldsymbol{x})=\left(\begin{array}{c}
\sin \left(\pi p_{1} x_{1}\right) \cos \left(\pi p_{2} x_{2}\right) \\
\cos \left(\pi p_{1} x_{1}\right) \sin \left(\pi p_{2} x_{2}\right) \\
0
\end{array}\right) .
\end{aligned}
$$

In a similar manner we define vectors $\boldsymbol{B}_{\boldsymbol{p}}^{(j)}$ to be tangential components of vector $\boldsymbol{A}_{\boldsymbol{p}}=\left(A_{p, 1}, A_{p, 2}, A_{p, 3}\right)$ on cube's boundaries $x_{j}=0$ or $x_{j}=1, j=1,2,3$,

$$
\boldsymbol{B}_{\boldsymbol{p}}^{(1)}=\left(\begin{array}{c}
0 \\
A_{p, 2} \\
A_{p, 3}
\end{array}\right), \quad \boldsymbol{B}_{\boldsymbol{p}}^{(2)}=\left(\begin{array}{c}
A_{p, 1} \\
0 \\
A_{p, 3}
\end{array}\right), \quad \boldsymbol{B}_{\boldsymbol{p}}^{(3)}=\left(\begin{array}{c}
A_{p, 1} \\
A_{p, 2} \\
0
\end{array}\right) .
$$

The corresponding vector $\boldsymbol{A}_{\boldsymbol{p}}$ in this case has two polarizations $\boldsymbol{A}_{\boldsymbol{p}+}$ and $\boldsymbol{A}_{\boldsymbol{p}-}$. However, on the cube's surface one of its components vanishes and we have

$$
\boldsymbol{A}_{\boldsymbol{p}+}=\boldsymbol{B}_{\boldsymbol{p}+}^{(j)} \quad \text { or } \quad \boldsymbol{A}_{\boldsymbol{p}-}=\boldsymbol{B}_{\boldsymbol{p}-}^{(j)} \quad \text { for } p_{j}=0 .
$$


Let us show that two-dimensional vectors $\boldsymbol{B}_{\boldsymbol{p}+}^{(j)}$ and $\boldsymbol{B}_{\boldsymbol{p}-}^{(j)}$ are linearly independent by the example of $j=1$. In this case

$$
\boldsymbol{B}_{\boldsymbol{p}+}^{(1)}=\left(\begin{array}{c}
0 \\
A_{p+, 2} \\
A_{p+, 3}
\end{array}\right), \quad \boldsymbol{B}_{\boldsymbol{p}-}^{(1)}=\left(\begin{array}{c}
0 \\
A_{p-, 2} \\
A_{p-, 3}
\end{array}\right)
$$

Suppose that this is not so and there exist two complex numbers $\alpha_{+}$and $\alpha_{-}$, not both zero, such that

$$
\alpha_{+}\left(\begin{array}{c}
A_{p+, 2} \\
A_{p+, 3}
\end{array}\right)+\alpha_{-}\left(\begin{array}{c}
A_{p-, 2} \\
A_{p-, 3}
\end{array}\right)=0
$$

Multiplying the first and the second rows by $p_{2}$ and $p_{3}$, respectively, and combining these equations with the divergence-free condition (2.13)

$$
p_{1} A_{p \pm, 1}+p_{2} A_{p \pm, 2}+p_{3} A_{p \pm, 3}=0,
$$

which holds for either $\boldsymbol{A}_{\boldsymbol{p}+}$ and $\boldsymbol{A}_{\boldsymbol{p}-}$, we obtain

$$
\alpha_{+} p_{1} A_{p+, 1}+\alpha_{-} p_{1} A_{p-, 1}=0 .
$$

Since $\boldsymbol{p} \in \mathcal{P}_{ \pm}$and $p_{1}>0$, (3.15) together with ( 3.16) imply that $\alpha_{+} \boldsymbol{A}_{\boldsymbol{p}+}+\alpha_{-} \boldsymbol{A}_{\boldsymbol{p}-}=$ 0 , which clearly contradicts the original choice of $\boldsymbol{A}_{\boldsymbol{p}+}$ and $\boldsymbol{A}_{\boldsymbol{p}-}$ as linearly independent vectors. Thus, we have proven the following lemma.

Lemma 3.1. For every $j=1,2,3$ and fixed $\boldsymbol{p}$ from $\mathcal{P}_{ \pm}$, polarization vectors $\boldsymbol{A}_{\boldsymbol{p}+}^{(j)}$ and $\boldsymbol{A}_{\boldsymbol{p}-}^{(j)}$ are linearly independent on the boundary of the cavity.

Case 2. If the index $\boldsymbol{p} \in \mathcal{P}_{0}$ has exactly one zero entry $p_{j}$, the corresponding component $A_{p, j}$ of the polarization vector $\boldsymbol{A}_{p}$ vanishes. On the faces $x_{j}=0$ or $x_{j}=1, j=1,2,3$, such polarization vectors $\boldsymbol{B}_{\boldsymbol{p}}^{(j)}$ have the form

$$
\boldsymbol{B}_{0, p_{2}, p_{3}}^{(1)}=\left(\begin{array}{c}
0 \\
A_{p, 2} \\
A_{p, 3}
\end{array}\right), \quad \boldsymbol{B}_{p_{1}, 0, p_{3}}^{(2)}=\left(\begin{array}{c}
A_{p, 1} \\
0 \\
A_{p, 3}
\end{array}\right), \quad \boldsymbol{B}_{p_{1}, p_{2}, 0}^{(3)}=\left(\begin{array}{c}
A_{p, 1} \\
A_{p, 2} \\
0
\end{array}\right) .
$$

However, on the faces $x_{k}=0$ or $x_{k}=1, k=1,2,3$, where $k \neq j$, the polarization vector $\boldsymbol{B}_{p}^{(k)}$ will have two zero components. It is convenient to scale the vectors by a factor of $\sqrt{2}$ :

$$
\begin{aligned}
& \boldsymbol{B}_{p_{1}, 0, p_{3}}^{(1)}=\left(\begin{array}{c}
0 \\
0 \\
\sqrt{2} A_{p, 3}
\end{array}\right), \quad \boldsymbol{B}_{p_{1}, p_{2}, 0}^{(1)}=\left(\begin{array}{c}
0 \\
\sqrt{2} A_{p, 2} \\
0
\end{array}\right), \quad \boldsymbol{B}_{p_{1}, p_{2}, 0}^{(2)}=\left(\begin{array}{c}
\sqrt{2} A_{p, 1} \\
0 \\
0
\end{array}\right), \\
& \boldsymbol{B}_{0, p_{2}, p_{3}}^{(2)}=\left(\begin{array}{c}
0 \\
0 \\
\sqrt{2} A_{p, 3}
\end{array}\right), \quad \boldsymbol{B}_{0, p_{2}, p_{3}}^{(3)}=\left(\begin{array}{c}
\sqrt{2} A_{p, 1} \\
\sqrt{2} A_{p, 2} \\
0 \\
0
\end{array}\right), \quad \boldsymbol{B}_{p_{1}, 0, p_{3}}^{(3)}=\left(\begin{array}{c}
\sqrt{2)}
\end{array}\right) .
\end{aligned}
$$

Components of the eigenmodes' projections $\boldsymbol{\Theta}_{\boldsymbol{p}}^{(k)}(\boldsymbol{x})$ in this case have, analogous to (3.18), the form 


$$
\begin{aligned}
& \Theta_{p_{1}, 0, p_{3}}^{(1)}=\left(\begin{array}{c}
0 \\
0 \\
\sin \pi p_{3} x_{3}
\end{array}\right), \Theta_{p_{1}, p_{2}, 0}^{(1)}=\left(\begin{array}{c}
0 \\
\sin \pi p_{2} x_{2} \\
0
\end{array}\right), \Theta_{p_{1}, p_{2}, 0}^{(2)}=\left(\begin{array}{c}
\sin \pi p_{1} x_{1} \\
0 \\
0
\end{array}\right), \\
& \Theta_{0, p_{2}, p_{3}}^{(2)}=\left(\begin{array}{c}
0 \\
0 \\
\sin \pi p_{3} x_{3}
\end{array}\right), \Theta_{0, p_{2}, p_{3}}^{(3)}=\left(\begin{array}{c}
\sin \pi p_{1} x_{1} \\
0 \\
0 \\
\sin \pi p_{2} x_{2} \\
0
\end{array}\right), \Theta_{p_{1}, 0, p_{3}}^{(3)}=\left(\begin{array}{c}
{ }_{0}
\end{array}\right) .
\end{aligned}
$$

4. Matrix form of the Maxwell operator. To represent operator $M(\boldsymbol{k}, \eta)$ (3.10) in matrix form we need to establish a one-to-one correspondence between the index $\boldsymbol{p}$ of the eigenvalue (2.6) and its eigenmode. To this end, we will count twice the indices $\boldsymbol{p}$ of multiplicity two from the set $\mathcal{P}_{ \pm}$. The new set is denoted by $\check{\mathcal{P}}_{ \pm}$, and in what follows we will use the index $\check{\boldsymbol{p}} \in \check{\mathcal{P}}$ of multiplicity one, where

$$
\check{\mathcal{P}}=\check{\mathcal{P}}_{ \pm} \cup \mathcal{P}_{0} .
$$

We introduce next the eigenmode expansion of a vector field $\boldsymbol{F}(\boldsymbol{x}) \in L_{2}\left(Q_{0}\right)$

$$
\boldsymbol{F}(\boldsymbol{x})=\sum_{\check{p} \in \check{\mathcal{P}}} F_{\breve{p}} \Psi_{\check{p}}(\boldsymbol{x}) .
$$

Transformation (4.2) of the function $\boldsymbol{G}(\boldsymbol{x}) \in L_{2}\left(Q_{0}\right)$ to the sequence $\left\{G_{\check{p}}\right\} \in l_{2}(\check{\mathcal{P}})$ is unitary, i.e.,

$$
\int_{Q_{0}} \boldsymbol{F}(\boldsymbol{x}) \cdot \overline{\boldsymbol{G}(\boldsymbol{x})} d \boldsymbol{x}=\sum_{\breve{p} \in \check{\mathcal{P}}} F_{\breve{p}} \bar{G}_{\check{p}} .
$$

Matrix representation $\mathscr{M}_{0}$ of the operator $M_{0}$ in the eigenbasis $\left\{\boldsymbol{\Psi}_{\check{p}}(\boldsymbol{x})\right\}$ has diagonal form

$$
\mathscr{M}_{0}=\operatorname{diag}\left(\Lambda_{1}, \Lambda_{2}, \ldots\right),
$$

where $\Lambda_{i}, i=1,2, \ldots$, is a diagonal matrix with $\xi_{i}=\pi^{2} \check{\boldsymbol{p}}_{i}^{2}(2.6)$ on its diagonal. The order of the block $\Lambda_{i}$ equals the multiplicity of the eigenvalue $\xi_{i}$.

Calculation of the elements $\left[\mathscr{M}_{1}\right]_{\breve{p} \breve{q}}$ of Hermitian matrix $\mathscr{M}_{1}$ corresponding to the operator $M_{1}(\boldsymbol{k})$ yields

$$
\begin{aligned}
& {\left[\mathscr{M}_{1}\right]_{\check{\boldsymbol{p}} \breve{\boldsymbol{q}}}=\frac{\left[1-(-1)^{\check{p}_{1}} e^{i k_{1}}\right]\left[1-(-1)^{\check{q}_{1}} e^{-i k_{1}}\right]}{4}\left\{A_{\check{p}, 2} A_{\check{q}_{2}, 2}\left(\delta_{\check{p}_{2}, \check{q}_{2}}-\delta_{\check{p}_{2}, 0} \delta_{\check{q}_{2}, 0}\right)\right.} \\
& \left.\left(\delta_{\check{p}_{3}, \check{q}_{3}}+\delta_{\check{p}_{3}, 0} \delta_{\check{q}_{3}, 0}\right)+A_{\check{p}, 3} A_{\check{q}_{3}, 3}\left(\delta_{\check{p}_{2}, \check{q}_{2}}+\delta_{\check{p}_{2}, 0} \delta_{\check{q}_{2}, 0}\right)\left(\delta_{\check{p}_{3}, \check{q}_{3}}-\delta_{\check{p}_{3}, 0} \delta_{\check{q}_{3}, 0}\right)\right\} \\
& +\frac{\left[1-(-1)^{\check{p}_{2}} e^{i k_{2}}\right]\left[1-(-1)^{\check{q}_{2}} e^{-i k_{2}}\right]}{4}\left\{A_{\breve{p}, 1} A_{\breve{q}, 1}\left(\delta_{\check{p}_{1}, \check{q}_{1}}-\delta_{\check{p}_{1}, 0} \delta_{\check{q}_{1}, 0}\right)\right. \\
& \left.\left(\delta_{\check{p}_{3}, \check{q}_{3}}+\delta_{\check{p}_{3}, 0} \delta_{\check{q}_{3}, 0}\right)+A_{\check{p}, 3} A_{\check{q}_{3} 3}\left(\delta_{\check{p}_{1}, \check{q}_{1}}+\delta_{\check{p}_{1}, 0} \delta_{\check{q}_{1}, 0}\right)\left(\delta_{\check{p}_{3}, \check{q}_{3}}-\delta_{\check{p}_{3}, 0} \delta_{\check{q}_{3}, 0}\right)\right\} \\
& +\frac{\left[1-(-1)^{\check{p}_{3}} e^{i k_{3}}\right]\left[1-(-1)^{\check{q}_{3}} e^{-i k_{3}}\right]}{4}\left\{A_{\check{p}, 1} A_{\check{q}_{1}, 1}\left(\delta_{\check{p}_{1}, \check{q}_{1}}-\delta_{\check{p}_{1}, 0} \delta_{\check{q}_{1}, 0}\right)\right. \\
& \left.\left(\delta_{\check{p}_{2}, \check{q}_{2}}+\delta_{\check{p}_{2}, 0} \delta_{\check{q}_{2}, 0}\right)+A_{\check{p}, 2} A_{\check{q}_{2}}\left(\delta_{\check{p}_{1}, \check{q}_{1}}+\delta_{\check{p}_{1}, 0} \delta_{\check{q}_{1}, 0}\right)\left(\delta_{\check{p}_{2}, \breve{q}_{2}}-\delta_{\check{p}_{2}, 0} \delta_{\check{q}_{2}, 0}\right)\right\},
\end{aligned}
$$

where $\delta_{p, q}$ is the Kronecker delta. 
Expression (4.6) is simplified if $\check{\boldsymbol{p}}$ and $\check{\boldsymbol{q}}$ do not contain zero components. Then

$$
\left|A_{\check{\boldsymbol{p}}}\right|^{2}=\left|A_{\check{\boldsymbol{q}}}\right|^{2}=8
$$

and

$\left[\mathscr{M}_{1}\right]_{\check{\boldsymbol{p}} \check{\boldsymbol{q}}}=\frac{1}{4} \sum_{j=1}^{3}\left[1-(-1)^{\check{p}_{j}} e^{i k_{j}}\right]\left[1-(-1)^{\check{q}_{j}} e^{-i k_{j}}\right] \frac{\delta_{\check{p}_{1}, \check{q}_{1}} \delta_{\check{p}_{2}, \check{q}_{2}} \delta_{\check{p}_{3}, \check{q}_{3}}}{\delta_{\check{p}_{j}, \breve{q}_{j}}}\left(A_{\breve{p}} \cdot A_{\check{q}}-A_{\check{p}, j} A_{\check{q}, j}\right)$.

In particular, when $\check{\boldsymbol{p}}=\check{\boldsymbol{q}}$ diagonal elements of $\mathscr{M}_{1}$ have the form

$$
\left[\mathscr{M}_{1}\right]_{\breve{\boldsymbol{p}} \check{\boldsymbol{p}}}=\frac{1}{2} \sum_{j=1}^{3}\left[1-(-1)^{\check{p}_{j}} \cos k_{i}\right]\left(8-A_{\check{p}, j}^{2}\right)
$$

Alternatively, if among components of the vectors $\check{\boldsymbol{p}}$ or $\check{\boldsymbol{q}}$ there is zero, say, $\check{p}_{1}=0$, then

$$
\left|\boldsymbol{A}_{\breve{p}}\right|^{2}=A_{\tilde{p}, 2}^{2}+A_{\check{p}, 3}^{2}=4
$$

and for diagonal elements (4.6) gives

$$
\left[\mathscr{M}_{1}\right]_{\check{\boldsymbol{p}} \check{\boldsymbol{p}}}=6-2 \cos k_{1}-(-1)^{\check{p}_{2}} A_{\check{p}, 3}^{2} \cos k_{2}-(-1)^{\check{p}_{3}} A_{\check{p}, 2}^{2} \cos k_{3}
$$

From inspection of (4.6) one can derive the following properties of $\mathscr{M}_{1}$ and its elements.

(i) $\mathscr{M}_{1}$ is a sparse matrix. For fixed $\check{\boldsymbol{p}}$ the element $\left[\mathscr{M}_{1}\right]_{\check{\boldsymbol{p}} \check{\boldsymbol{q}}}$ is nonzero if and only if $(\check{\boldsymbol{p}}-\check{\boldsymbol{q}})$ is parallel to $\boldsymbol{e}_{i}, i=1,2,3$, i.e., $\check{\boldsymbol{q}}$ belongs to the line passing through $\check{\boldsymbol{p}}$ and parallel to one of the coordinate axes.

(ii) Any nonzero matrix element $\left[\mathscr{M}_{1}\right]_{\check{\boldsymbol{p}} \check{\boldsymbol{q}}}$ vanishes when $\boldsymbol{k}=\frac{\pi}{2}\left(1-(-1)^{\check{p}_{1}}, 1-\right.$ $\left.(-1)^{\check{p}_{2}}, 1-(-1)^{\check{p}_{3}}\right)$ or $\boldsymbol{k}=\frac{\pi}{2}\left(1-(-1)^{\check{q}_{1}}, 1-(-1)^{\check{q}_{2}}, 1-(-1)^{\check{q}_{3}}\right)$.

(iii) $\max _{\boldsymbol{k}}\left|\left[\mathscr{M}_{1}\right]_{\check{\boldsymbol{p}} \check{\boldsymbol{p}}}\right|=4\left(4-\delta_{\check{p}_{1}, 0}-\delta_{\check{p}_{2}, 0}-\delta_{\check{p}_{3}, 0}\right)$. If $\check{\boldsymbol{p}} \in \mathcal{P}_{ \pm}$, i.e., vector $A_{\check{\boldsymbol{p}}}$ admits two polarizations (no zeros among $\check{p}_{i}, i=1,2,3$ ), then $\max _{\boldsymbol{k}}\left|\left[\mathscr{M}_{1}\right]_{\check{p} \check{p}}\right|=16$. Otherwise $\max _{\boldsymbol{k}}\left|\left[\mathscr{M}_{1}\right]_{\check{p} \check{p}}\right|=12$.

(iv) Maximum value of the quadratic form $\left(M_{1}(\boldsymbol{k}) \boldsymbol{\Psi}_{\breve{p}}, \boldsymbol{\Psi}_{\check{\boldsymbol{p}}}\right)$ is attained on the quasi momentum vector $\boldsymbol{k}_{\max }=\frac{\pi}{2}\left(1+(-1)^{\check{p}_{1}}, 1+(-1)^{\check{p}_{2}}, 1+(-1)^{\check{p}_{3}}\right)$, and if $\check{\boldsymbol{p}} \in \mathcal{P}_{ \pm}$, then $\max \left(M_{1}(\boldsymbol{k}) \boldsymbol{\Psi}_{\check{\boldsymbol{p}}}, \boldsymbol{\Psi}_{\check{\boldsymbol{p}}}\right)=2\left|\boldsymbol{A}_{\breve{p}}\right|^{2}=16$. Otherwise, when vector $A_{\breve{\boldsymbol{p}}}$ has the sole polarization then $\max \left(M_{1}(\boldsymbol{k}) \boldsymbol{\Psi}_{\check{\boldsymbol{p}}}, \boldsymbol{\Psi}_{\check{\boldsymbol{p}}}\right)=3\left|\boldsymbol{A}_{\check{\boldsymbol{p}}}\right|^{2}=12$. These maximum values define the upper bounds of perturbed eigenvalues in the linear approximation.

The entries of $\mathscr{M}_{1}$ have natural three-dimensional indices. In order to rearrange them in a two-dimensional matrix we enumerate three-dimensional vector-indices $\check{\boldsymbol{p}}$ in ascending order of $|\check{\boldsymbol{p}}|$. Thus, calculation of the eigenvalues of the operator $M(\boldsymbol{k}, \eta)$ (3.10) is reduced to the calculation of the eigenvalues of the infinite matrix $\mathscr{M}_{0}+\eta \mathscr{M}_{1}$. 


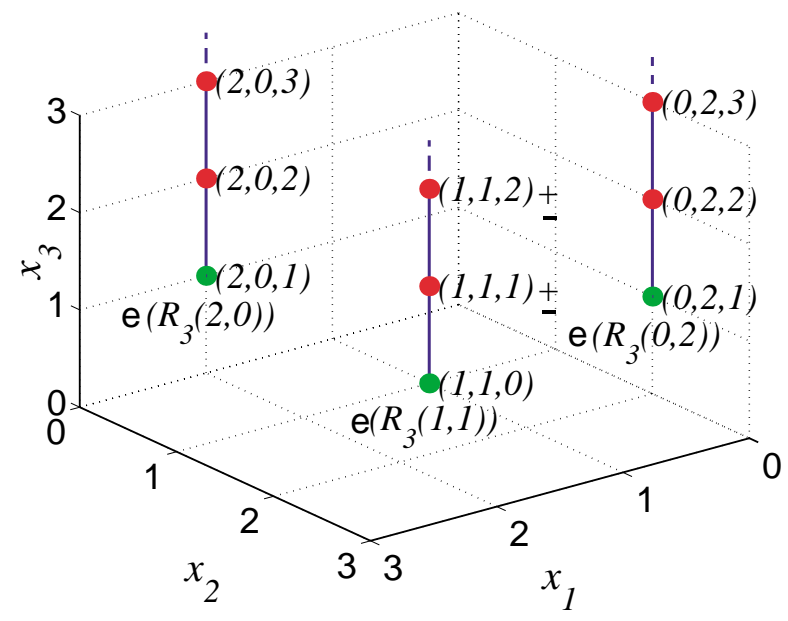

FIG. 5.1. Integer-valued indices of eigenvalues combined into rays in the $x_{3}$ direction. The sign \pm denotes indices of multiplicity two. $\mathrm{e}\left(R_{3}(2,0)\right), \mathrm{e}\left(R_{3}(1,1)\right), \mathrm{e}\left(R_{3}(0,2)\right)$ denote end points of the rays.

\section{Relative compactness of the perturbation operator.}

5.1. Projection representation. The set of the indices on which matrix elements $\left[\mathscr{M}_{1}\right]_{\breve{p} \breve{q}}$ do not vanish makes up rays along the coordinate axes. We denote the rays by

$$
\begin{aligned}
& R_{1}\left(p_{2}, p_{3}\right)=\bigcup_{p_{1}=\delta\left(p_{2}, p_{3}\right)}^{\infty}\left(p_{1}, p_{2}, p_{3}\right), R_{2}\left(p_{1}, p_{3}\right)=\bigcup_{p_{2}=\delta\left(p_{1}, p_{3}\right)}^{\infty}\left(p_{1}, p_{2}, p_{3}\right), \\
& R_{3}\left(p_{1}, p_{2}\right)=\bigcup_{p_{3}=\delta\left(p_{1}, p_{2}\right)}^{\infty}\left(p_{1}, p_{2}, p_{3}\right),
\end{aligned}
$$

where $p_{1}, p_{2}, p_{3}$ are nonnegative integers, such that at most one of them can be zero, and

$$
\delta(s, t)= \begin{cases}0 & \text { if } s, t>0 \\ 1 & \text { if } s=0 \text { or } t=0\end{cases}
$$

The end point of the ray $R_{j}(s, t)$, denoted by $\mathrm{e}\left(R_{j}(s, t)\right)$, lies in the plane $x_{j}=0$ if both $s$ and $t$ are positive (Figure 5.1). Thus, index $j=\operatorname{dir}\left(R_{j}(s, t)\right)$ shows the direction $x_{j}$ of the ray $R_{j}(s, t), j=1,2,3$. Also the ray whose points are counted, taking into account their multiplicity, we denote by $\check{R}_{j}(s, t)$.

For computation of the quadratic form (3.11) it is convenient to introduce new notation for the tangential components of the eigenmodes (3.13) on the faces $x_{j}=0$ or $x_{j}=1, j=1,2,3$. We denote

$$
\boldsymbol{\Theta}_{p}^{(1)}(\boldsymbol{x})=\boldsymbol{\Theta}_{p_{2} p_{3}}^{(1)}(\boldsymbol{x}), \quad \boldsymbol{\Theta}_{\boldsymbol{p}}^{(2)}(\boldsymbol{x})=\boldsymbol{\Theta}_{p_{1} p_{3}}^{(2)}(\boldsymbol{x}), \quad \boldsymbol{\Theta}_{\boldsymbol{p}}^{(3)}(\boldsymbol{x})=\boldsymbol{\Theta}_{p_{1} p_{2}}^{(3)}(\boldsymbol{x})
$$


Then the boundary values of $\boldsymbol{F}(\boldsymbol{x})$ (4.2) take the form

$$
\begin{aligned}
\left.\boldsymbol{F}(\boldsymbol{x})\right|_{x_{j}=0} & =\sum_{(s, t) \in \mathbb{N}_{+}^{2}} \sum_{\check{\boldsymbol{p}} \in \check{R}_{j}(s, t)} F_{\breve{\boldsymbol{p}}} \boldsymbol{A}_{\check{\boldsymbol{p}}} \star \boldsymbol{\Theta}_{s t}^{(j)}(\boldsymbol{x}), \\
\left.\boldsymbol{F}(\boldsymbol{x})\right|_{x_{j}=1} & =\sum_{(s, t) \in \mathbb{N}_{+}^{2}} \sum_{\breve{\boldsymbol{p}} \in \check{R}_{j}(s, t)}(-1)^{p_{j}} F_{\breve{\boldsymbol{p}}} \boldsymbol{A}_{\breve{\boldsymbol{p}}} \star \boldsymbol{\Theta}_{s t}^{(j)}(\boldsymbol{x}), \quad j=1,2,3,
\end{aligned}
$$

and

$$
\left.\boldsymbol{F}(\boldsymbol{x})\right|_{x_{j}=0}-\left.e^{i k_{j}} \boldsymbol{F}(\boldsymbol{x})\right|_{x_{j}=1}=\sum_{(s, t) \in \mathbb{N}_{+}^{2}} \sum_{\check{\boldsymbol{p}} \in \check{R}_{j}(s, t)}\left(1-(-1)^{p_{j}} e^{i k_{j}}\right) F_{\check{\boldsymbol{p}}} \boldsymbol{A}_{\check{\boldsymbol{p}}} \star \boldsymbol{\Theta}_{s t}^{(j)}(\boldsymbol{x}),
$$

where $\mathbb{N}_{+}^{2}$ is the set of nonnegative integers, not both zero.

Functions $\boldsymbol{\Theta}_{s t}^{(j)}(\boldsymbol{x})$ are orthogonal for fixed $j$ on the surface of the unit cube. For the integrals (3.11) of nonzero components of $\boldsymbol{\Theta}_{s t, m}^{(j)}(\boldsymbol{x})$ we have (cf. (3.11))

$$
\begin{aligned}
\left(\boldsymbol{\Theta}_{s t, m}^{(j)}(\boldsymbol{x}), \boldsymbol{\Theta}_{s t, m}^{(j)}(\boldsymbol{x})\right) & =\int_{\gamma_{j}}\left|\boldsymbol{\Theta}_{s t, m}^{(j)}(\boldsymbol{x})\right|^{2} d S \\
& =\left\{\begin{array}{ll}
\frac{1}{4} & \text { if } s, t>0, \\
\frac{1}{2} & \text { if } s=0 \text { or } t=0,
\end{array} \neq m, \quad j, m=1,2,3 .\right.
\end{aligned}
$$

We calculate quadratic form (3.11) of the operator $M_{1}$ on the eigenfunction expansion of $\boldsymbol{F}(\boldsymbol{x})$ (4.2) to estimate its norm. From (5.5) we have

$$
\begin{aligned}
& \left(M_{1}(\boldsymbol{k}) \boldsymbol{F}, \boldsymbol{F}\right)=\sum_{j=1}^{3} \int_{\gamma_{j}} \sum_{(s, t) \in \mathbb{N}_{+}^{2}}\left|\sum_{\check{\boldsymbol{p}} \in \check{R}_{j}(s, t)}\left(1-(-1)^{p_{j}} e^{i k_{j}}\right) F_{\breve{\boldsymbol{p}}} \boldsymbol{A}_{\check{\boldsymbol{p}}} \star \boldsymbol{\Theta}_{s t}^{(j)}(\boldsymbol{x})\right|^{2} d S \\
& =\frac{1}{4} \sum_{j=1}^{3} \sum_{(s, t) \in \mathbb{N}_{+}^{2}} \sum_{\check{\boldsymbol{p}} \in \check{R}_{j}(s, t)}\left|\alpha_{\boldsymbol{p} p}^{(j)} F_{\breve{\boldsymbol{p}}} \boldsymbol{B}_{\check{\boldsymbol{p}}}^{(j)}(\boldsymbol{k})\right|^{2}=\frac{1}{4} \sum_{\check{R} \in \mathcal{R}} \sum_{\check{\boldsymbol{p}} \in \check{R}}\left|\alpha_{\boldsymbol{p} p}^{(\operatorname{dir}(\check{R}))} F_{\breve{\boldsymbol{p}}} \boldsymbol{B}_{\check{\boldsymbol{p}}}^{(\operatorname{dir}(\check{R}))}(\boldsymbol{k})\right|^{2},
\end{aligned}
$$

where

$$
\begin{aligned}
\boldsymbol{B}_{\ddot{\boldsymbol{p}}}^{(j)}(\boldsymbol{k}) & =\left(1-(-1)^{p_{j}} e^{i k_{j}}\right) \boldsymbol{B}_{\ddot{\boldsymbol{p}}}^{(j)}, \\
\alpha_{\boldsymbol{p} q}^{(j)} & = \begin{cases}\delta_{p_{2}, q_{2}} \delta_{p_{3}, q_{3}}, & j=1, \\
\delta_{p_{1}, q_{1}} \delta_{p_{3}, q_{3}}, & j=2, \\
\delta_{p_{1}, q_{1}} \delta_{p_{2}, q_{2}}, & j=3,\end{cases}
\end{aligned}
$$

and $\mathcal{R}=\bigcup_{j=1}^{3} R_{j}$ denotes the set of all rays (5.1) in the first octant.

For further analysis it is constructive to rewrite representation (5.7) in the projection form. To this end, we introduce an infinite-dimensional vectors $\mathscr{B}$ whose components are three-dimensional vectors $\boldsymbol{B}_{\check{\boldsymbol{p}}}^{(j)}(\boldsymbol{k})$. If we sort points $\check{\boldsymbol{p}}$ in ascending order of $|\check{\boldsymbol{p}}|$ taking into account their multiplicity, then (5.7) suggests to represent $\mathscr{B}$ as the following sum:

$$
\mathscr{B}(\boldsymbol{k})=\mathscr{B}^{(1)}(\boldsymbol{k})+\mathscr{B}^{(2)}(\boldsymbol{k})+\mathscr{B}^{(3)}(\boldsymbol{k}),
$$


where

$$
\begin{aligned}
\mathscr{B}^{(j)}(\boldsymbol{k})= & \left\{\boldsymbol{B}_{0,1,1}^{(j)}(\boldsymbol{k}), \boldsymbol{B}_{1,0,1}^{(j)}(\boldsymbol{k}), \boldsymbol{B}_{1,1,0}^{(j)}(\boldsymbol{k}), \boldsymbol{B}_{1,1,1+}^{(j)}(\boldsymbol{k}), \boldsymbol{B}_{1,1,1-}^{(j)}(\boldsymbol{k}), \boldsymbol{B}_{0,2,1}^{(j)}(\boldsymbol{k}),\right. \\
& \left.\boldsymbol{B}_{0,1,2}^{(j)}(\boldsymbol{k}), \boldsymbol{B}_{2,0,1}^{(j)}(\boldsymbol{k}), \boldsymbol{B}_{1,0,2}^{(j)}(\boldsymbol{k}), \boldsymbol{B}_{2,1,0}^{(j)}(\boldsymbol{k}), \ldots\right\}, \quad j=1,2,3 .
\end{aligned}
$$

In a similar manner we introduce infinite-dimensional vector $\mathscr{F}$ whose scalar components are the Fourier coefficients $F_{\breve{p}}$ of the function $\boldsymbol{F}(\boldsymbol{x})(4.2)$

$$
\mathscr{F}=\left\{F_{0,1,1}, F_{1,0,1}, F_{1,1,0}, F_{1,1,1+}, F_{1,1,1-}, F_{0,2,1}, F_{0,1,2}, F_{2,0,1}, F_{1,0,2}, F_{2,1,0}, \ldots\right\} .
$$

Quadratic form (5.7) is given now by

$$
\left(M_{1}(\boldsymbol{k}) \boldsymbol{F}, \boldsymbol{F}\right)=\frac{1}{4} \sum_{\check{R} \in \mathcal{R}}|(\mathscr{F}, \mathscr{B}(\boldsymbol{k}))|^{2} .
$$

5.2. Properties of the projection representation. Representations (5.7) and (5.13) allow us to prove efficiently that the perturbation operator $M_{1}(\boldsymbol{k})(3.11)$ is relatively compact to $M_{0}(2.16)$, i.e., the operator $\mathfrak{M}(\boldsymbol{k})$ is compact. The norm of the operator $\mathfrak{M}(\boldsymbol{k})$ (3.12) can be estimated through its quadratic form

$$
(\mathfrak{M}(\boldsymbol{k}) \boldsymbol{F}, \boldsymbol{F})=\frac{1}{4} \sum_{j=1}^{3} \sum_{(s, t) \in \mathbb{N}_{+}^{2}} \sum_{\check{\boldsymbol{p}} \in \check{R}_{j}(s, t)}\left|F_{\breve{\boldsymbol{p}}} \boldsymbol{b}_{\check{\boldsymbol{p}}}^{(j)}(\boldsymbol{k})\right|^{2}=\frac{1}{4} \sum_{j=1}^{3} \sum_{\check{R} \in \mathcal{R}}\left|\left(\mathscr{F}, \boldsymbol{b}^{(j)}(\boldsymbol{k})\right)\right|^{2},
$$

where

$$
\boldsymbol{b}^{(j)}(\boldsymbol{k})=\left\{\alpha_{\boldsymbol{p} p}^{(j)} \boldsymbol{b}_{\check{\boldsymbol{p}}}^{(j)}(\boldsymbol{k})\right\}, \quad \boldsymbol{b}_{\check{\boldsymbol{q}}}^{(j)}(k)=\left\{\begin{array}{cc}
\boldsymbol{B}_{\check{\boldsymbol{p}}}^{(j)}(\boldsymbol{k}) /|\boldsymbol{p}| & \text { if } \check{\boldsymbol{q}}=\check{\boldsymbol{p}}\left(\check{R}_{j}\right) \\
0 & \text { otherwise }
\end{array}\right.
$$

From (5.14) it follows that operator $\mathfrak{M}(\boldsymbol{k})$ is the sum of projections on vectors $\boldsymbol{b}^{(j)}(\boldsymbol{k})$

$$
\mathfrak{M}(\boldsymbol{k})=\frac{1}{4} \sum_{j=1}^{3} \sum_{\check{R} \in \mathcal{R}} P\left(\boldsymbol{b}^{(j)}(\boldsymbol{k})\right), \quad \text { with } P(\boldsymbol{b}) \boldsymbol{a}=(\boldsymbol{b}, \boldsymbol{a}) \boldsymbol{b} .
$$

Since $\boldsymbol{b}$ is not a unit vector $\|P\|=\|\boldsymbol{b}\|^{2}$.

Quadratic form (5.14) yields matrix elements of the operator $\mathfrak{M}(\boldsymbol{k})$

$$
\begin{aligned}
{[\mathfrak{M}(\boldsymbol{k})]_{\check{\boldsymbol{p}} \check{\boldsymbol{q}}}=} & \frac{1}{4} \sum_{j=1}^{3} \alpha_{\boldsymbol{p} q}^{(j)} \boldsymbol{b}_{\check{\boldsymbol{p}}}^{(j)}(\boldsymbol{k}) \overline{\boldsymbol{b}}_{\check{\boldsymbol{q}}}^{(j)}(k)=\frac{1}{4|\boldsymbol{p} \| \boldsymbol{q}|} \sum_{j=1}^{3} \alpha_{\boldsymbol{p q}}^{(j)}\left[1-(-1)^{p_{j}} e^{i k_{j}}\right] \\
& {\left[1-(-1)^{q_{j}} e^{-i k_{j}}\right]\left(\boldsymbol{B}_{\tilde{\boldsymbol{p}}}^{(j)}, \boldsymbol{B}_{\check{\boldsymbol{q}}}^{(j)}\right), \quad \check{\boldsymbol{p}}, \check{\boldsymbol{q}} \in \check{R} . }
\end{aligned}
$$

For example, if $\check{\boldsymbol{p}}=(0,1,1)$ and $\check{\boldsymbol{q}}=(1,1,1+)$, then

$$
\begin{aligned}
{[\mathfrak{M}(\boldsymbol{k})]_{14} } & =\frac{1}{4 \sqrt{6}}\left[1-e^{i k_{1}}\right]\left[1+e^{-i k_{1}}\right]\left(A_{p, 2} A_{q, 2}+A_{p, 3} A_{q, 3}\right) \\
& =-\frac{i \sin k_{1}}{2 \sqrt{6}}\left(A_{p, 2} A_{q, 2}+A_{p, 3} A_{q, 3}\right) .
\end{aligned}
$$


In the case when $\check{\boldsymbol{p}}=\check{\boldsymbol{q}}=(0,1,1)$ we obtain

$$
\begin{aligned}
{[\mathfrak{M}(\boldsymbol{k})]_{11}=} & \frac{1}{4 \sqrt{2}}\left\{\left[1-e^{i k_{1}}\right]\left[1-e^{-i k_{1}}\right]\left(A_{p, 2}^{2}+A_{p, 3}^{2}\right)+2\left[1+e^{i k_{2}}\right]\left[1+e^{-i k_{2}}\right] A_{p, 3}^{2}\right. \\
& \left.+2\left[1+e^{i k_{3}}\right]\left[1+e^{-i k_{3}}\right] A_{p, 2}^{2}\right\}=\frac{1}{2 \sqrt{2}}\left\{\left(1-\cos k_{1}\right)\left(A_{p, 2}^{2}+A_{p, 3}^{2}\right)\right. \\
= & \left.2\left(1+\cos k_{2}\right) A_{p, 3}^{2}+2\left(1+\cos k_{3}\right) A_{p, 2}^{2}\right\} .
\end{aligned}
$$

Properties of the projection vectors $\boldsymbol{b}^{(j)}(\boldsymbol{k})$ are summarized in the following lemma.

Lemma 5.1. Vectors $\boldsymbol{b}^{(j)}\left(\check{R}_{j}, \boldsymbol{k}\right)$ associated with direction $x_{j}$ have the following properties:

(i) The only nonzero components of vector $\boldsymbol{b}^{(j)}\left(\check{R}_{j}, \boldsymbol{k}\right)$ are those whose vectorindex $\check{\boldsymbol{p}}$ belongs to $\check{R}_{j}$

$$
\left(\boldsymbol{e}_{\check{\boldsymbol{p}}}, \boldsymbol{b}^{(j)}\left(\check{R}_{j}, \boldsymbol{k}\right)\right)=\left\{\begin{array}{cc}
\boldsymbol{B}_{\check{\boldsymbol{p}}}^{(j)}(\boldsymbol{k}) /|\boldsymbol{p}| & \text { if } \boldsymbol{p} \in \check{R}_{j}, \\
0 & \text { if } \boldsymbol{p} \notin \check{R}_{j},
\end{array}\right.
$$

where $\boldsymbol{e}_{\check{\boldsymbol{p}}}$ are vectors of the standard basis in $l_{2}(\check{\mathcal{P}})$.

(ii) If two vectors $\boldsymbol{b}^{(j)}\left(\check{R}_{j}, \boldsymbol{k}\right)$ correspond to different nonintersecting rays, then they are orthogonal:

$$
\left(\boldsymbol{b}^{(m)}\left(\check{R}_{m}, \boldsymbol{k}\right), \boldsymbol{b}^{(n)}\left(\check{R}_{n}, \boldsymbol{k}\right)\right)=0 \text { if } \check{R}_{m} \cap \check{R}_{n}=\varnothing .
$$

(iii) Components of vector $\boldsymbol{b}^{(j)}\left(\check{R}_{j}, \boldsymbol{k}\right)$ are bounded by

$$
\left|\boldsymbol{b}_{\check{\boldsymbol{p}}}^{(j)}(k)\right| \leq \frac{4 \sqrt{2}}{|\boldsymbol{p}|} \quad \text { when } \boldsymbol{p} \in \check{R}_{j}
$$

(iv) Infinite-dimensional vectors $\boldsymbol{b}^{(j)}\left(\check{R}_{j}, \boldsymbol{k}\right)$ are bounded by

$$
\left\|\boldsymbol{b}^{(j)}\left(\check{R}_{j}, \boldsymbol{k}\right)\right\|_{l_{2}(\check{\mathcal{P}})}^{2} \leq 32\left(\frac{1}{\left|\mathrm{e}\left(\check{R}_{j}\right)\right|^{2}}+\frac{\pi}{\left|\mathrm{e}\left(\check{R}_{j}\right)\right|}\right) .
$$

Proof. Relations (5.20)-(5.22) readily follow from the definition (5.15) of vectors $\boldsymbol{b}_{\tilde{\boldsymbol{p}}}^{(j)}(k)$. To show (5.23) we make use of the inequality

$$
\sum_{n \geq m} \frac{1}{b^{2}+n^{2}} \leq \sum_{n \geq m} \int_{n-1}^{n} \frac{1}{b^{2}+t^{2}} d t=\int_{m-1}^{\infty} \frac{1}{b^{2}+t^{2}} d t=\frac{1}{b}\left(\frac{\pi}{2}-\arctan \frac{m-1}{b}\right),
$$

where $m \geq 1$ and $b \geq 1$. Then

$$
\sum_{n \geq 0} \frac{1}{b^{2}+n^{2}}=\frac{1}{b^{2}}+\sum_{n \geq 1} \frac{1}{b^{2}+n^{2}} \leq \frac{1}{b^{2}}+\frac{\pi}{2 b} .
$$

The latter, together with (5.22), gives the desired result. 
5.3. Relative compactness. Representations (5.14) and (5.16) suggest the following decomposition of the operator $\mathfrak{M}(\boldsymbol{k})$ along the rays $\check{R}_{j}, j=1,2,3$ :

$$
\mathfrak{M}(\boldsymbol{k})=\mathfrak{M}^{(1)}(\boldsymbol{k})+\mathfrak{M}^{(2)}(\boldsymbol{k})+\mathfrak{M}^{(3)}(\boldsymbol{k}),
$$

where

$$
\mathfrak{M}^{(j)}(\boldsymbol{k})=\frac{1}{4} \sum_{\check{R}_{j}} P\left(\boldsymbol{b}^{(j)}(\boldsymbol{k})\right), \quad j=1,2,3
$$

For numerical computation of spectrum we define the truncated counterparts of the operators $\mathfrak{M}(\boldsymbol{k})$ and $\mathfrak{M}^{(j)}(\boldsymbol{k})$ :

$$
\widetilde{\mathfrak{M}}_{s}(\boldsymbol{k})=\sum_{|\mathrm{e}(\check{R})| \leq s} \mathfrak{M}(\check{R}, \boldsymbol{k}), \quad \widetilde{\mathfrak{M}}_{s}^{(j)}(\boldsymbol{k})=\sum_{\left|\mathrm{e}\left(\check{R}_{j}\right)\right| \leq s} \mathfrak{M}^{(j)}\left(\check{R}_{j}, \boldsymbol{k}\right) .
$$

The basic properties of the operators $\mathfrak{M}(\boldsymbol{k}), \mathfrak{M}^{(j)}(\boldsymbol{k})$, and $\mathfrak{M}(\check{R}, \boldsymbol{k})$ are given in the following.

LEMMA 5.2.

(i) The rank of the operator $\mathfrak{M}(\check{R}, \boldsymbol{k})$ is either one or two depending on the number of projections in the sum (5.27). Its norm is bounded

$$
\|\mathfrak{M}(\check{R}, \boldsymbol{k})\| \leq\|\boldsymbol{b}(\check{R}, \boldsymbol{k})\|^{2} \leq 32\left(\frac{1}{|\mathrm{e}(\check{R})|^{2}}+\frac{\pi}{|\mathrm{e}(\check{R})|}\right) .
$$

(ii) All operators $\mathfrak{M}\left(\check{R}_{j}, \boldsymbol{k}\right)$ associated with one fixed direction $x_{j}, j=1,2,3$, commute. Moreover,

$$
\mathfrak{M}\left(\check{R}_{j}^{(1)}, \boldsymbol{k}\right) \mathfrak{M}\left(\check{R}_{j}^{(2)}, \boldsymbol{k}\right)=0 \quad \text { if } \operatorname{dir} \check{R}_{j}^{(1)}=\operatorname{dir} \check{R}_{j}^{(2)}, \check{R}_{j}^{(1)} \neq \check{R}_{j}^{(2)}
$$

Hence, representation (5.27) is block-diagonal with the block dimensions one or two.

(iii) Truncated operators $\widetilde{\mathfrak{M}}_{s}^{(j)}(\boldsymbol{k})$ converge in operator norm to $\mathfrak{M}^{(j)}(\boldsymbol{k})$ so that the following estimations hold:

$$
\begin{aligned}
\left\|\mathfrak{M}^{(j)}(\boldsymbol{k})-\widetilde{\mathfrak{M}}_{s}^{(j)}(\boldsymbol{k})\right\| & =\sup _{\check{R}_{j}:|\mathrm{e}(\check{R})| \leq s}\left\|\mathfrak{M}\left(\check{R}_{j}, \boldsymbol{k}\right)\right\| \leq \frac{32(1+\pi s)}{s^{2}} \\
\left\|\mathfrak{M}^{(j)}(\boldsymbol{k})\right\| & =\sup _{\check{R}_{j}}\left\|\mathfrak{M}\left(\check{R}_{j}, \boldsymbol{k}\right)\right\| \leq 32(1+\pi) .
\end{aligned}
$$

(iv) Operators $\widetilde{\mathfrak{M}}_{s}^{(j)}(\boldsymbol{k})$ and $\widetilde{\mathfrak{M}}_{s}(\boldsymbol{k})$ converge in the operator norm to $\mathfrak{M}^{(j)}(\boldsymbol{k})$ and $\mathfrak{M}(\boldsymbol{k})$, respectively, as $s \rightarrow \infty$ uniformly in $\boldsymbol{k} \in K$.

(v) Each operator $\widetilde{\mathfrak{M}}_{s}^{(j)}(\boldsymbol{k})$ has a finite rank and each operator $\mathfrak{M}^{(j)}(\boldsymbol{k})$ is compact. Therefore, $\mathfrak{M}(\boldsymbol{k})$ is also compact.

Proof. From (5.15)-(5.16) it follows that the rank of $\mathfrak{M}(\check{R}, \boldsymbol{k})$ is the same as the rank of the vector $\boldsymbol{B}_{\tilde{p}}^{(j)}(\boldsymbol{k})$, which can be only one or two. Then the norm estimate is given by (5.23).

Since projection vectors $\boldsymbol{b}^{(j)}(\boldsymbol{k})(5.16)$ corresponding to two nonintersecting rays $\check{R}_{j}^{(1)}$ and $\check{R}_{j}^{(2)}$ are orthogonal (5.21) so do the operators $\mathfrak{M}\left(\check{R}_{j}^{(1)}, \boldsymbol{k}\right)$ and $\mathfrak{M}\left(\check{R}_{j}^{(2)}, \boldsymbol{k}\right)$. 
Therefore, representation (5.27) is block-diagonal, and then (5.29) implies the estimates $(5.31)-(5.32)$. Convergence of the operators $\widetilde{\mathfrak{M}}_{s}^{(j)}(\boldsymbol{k})$ and $\widetilde{\mathfrak{M}}_{s}(\boldsymbol{k})$ follows straightforwardly from (5.31) and (5.26).

To show compactness of $\mathfrak{M}(\boldsymbol{k})$ observe that the number of rays $\check{R}$ for which $|\mathrm{e}(\check{R})| \leq s$ is finite, and hence operator $\widetilde{\mathfrak{M}}_{s}^{(j)}(\boldsymbol{k})$ defined by (5.28) is a finite sum of the operators of rank one or two. Thus $\widetilde{\mathfrak{M}}_{s}^{(j)}(\boldsymbol{k})$ must be of finite rank and, in particular, compact. Operator $\mathfrak{M}^{(j)}(\boldsymbol{k})$, as an operator norm limit, is therefore compact. This completes the proof of the lemma.

6. Computation of the spectrum. We employ perturbation techniques for the spectral analysis of matrix $\mathscr{M}$ given by (3.10)

$$
\mathscr{M}=\mathscr{M}_{0}+\eta \mathscr{M}_{1},
$$

where $\mathscr{M}_{0}$ is block-diagonal matrix and $\eta$ is a small parameter. For computation of highly degenerate spectrum of matrix $\mathscr{M}$ we use the approach outlined in [2]. Using a similarity transformation, we transform matrix $\mathscr{M}$ to a unitary equivalent matrix $\mathscr{X}$ that has the same block-diagonal form $\mathscr{X}=\operatorname{diag}\left\{X^{(1)}, X^{(2)}, \ldots\right\}$ as the unperturbed matrix $\mathscr{M}_{0}$ :

$$
\begin{gathered}
\mathscr{X}=e^{-\mathcal{S}(\eta)} \mathscr{M} e^{\mathcal{S}(\eta)}=\mathscr{M}_{0}+\eta \mathscr{X}_{1}+\eta^{2} \mathscr{X}_{2}+\cdots, \\
\mathcal{S}=\eta S_{1}+\eta^{2} S_{2}+\cdots,
\end{gathered}
$$

where matrices $S_{j}, j=1,2, \ldots$, do not depend on $\eta$ and $\mathscr{X}_{1}, \mathscr{X}_{2}, \ldots$ are diagonal block-matrices whose block dimensions coincide with those of matrix $\mathscr{M}_{0}$. The perturbation is then partitioned into the blocks corresponding to the distinct eigenvalues. Thus, instead of infinite-dimensional perturbation $\mathscr{M}_{1}$ we obtain an infinite series of independent finite-dimensional perturbations for each of the blocks. In the case of our problem the linear perturbation term $\mathscr{X}_{1}$ is calculated exactly. Calculation of the higher order matrices involves truncation error.

Matrix $\mathscr{X}$ can be written using Hausdorf's representation

$$
\mathscr{X}=e^{-\mathcal{S}} \mathscr{M} e^{\mathcal{S}}=\mathscr{M}+[\mathscr{M}, \mathcal{S}]+\frac{1}{2 !}[[\mathscr{M}, \mathcal{S}], \mathcal{S}]+\cdots,
$$

where the brackets denote the commutator of two matrices

$$
[\mathscr{A}, \mathscr{B}]=\mathscr{A} \mathscr{B}-\mathscr{B} \mathscr{A} \text {. }
$$

Substituting (6.2)-(6.3) into (6.4) and equating the terms of like power in $\eta$, we obtain the following expressions for the matrices $\mathscr{X}_{j}$ involved in (6.2):

$$
\begin{aligned}
& \mathscr{X}_{1}=\left[\mathscr{M}_{0}, S_{1}\right]+\mathscr{M}_{1}, \\
& \mathscr{X}_{2}=\left[\mathscr{M}_{0}, S_{2}\right]+\left[\mathscr{M}_{1}, S_{1}\right]+\frac{1}{2}\left[\left[\mathscr{M}_{0}, S_{1}\right], S_{1}\right], \ldots
\end{aligned}
$$

To find expansion (6.2) we represent matrix $\mathscr{M}_{1}$ as the sum of block-diagonal matrix $\overline{\mathscr{M}}_{1}$ and the matrix $\stackrel{\circ}{\mathscr{M}}_{1}$ whose diagonal blocks are zeros (so-called integrable matrix [2]):

$$
\mathscr{M}_{1}=\overline{\mathscr{M}}_{1}+\stackrel{\circ}{\mathscr{M}}_{1}
$$


Then

$$
\mathscr{X}_{1}=\left[\mathscr{M}_{0}, S_{1}\right]+\overline{\mathscr{M}}_{1}+\stackrel{\circ}{\mathscr{M}}_{1},
$$

and to get rid of the integrable part of $\mathscr{M}_{1}$ we take $S_{1}$ to be a solution of the equation

$$
\left[\mathscr{M}_{0}, S_{1}\right]=-\stackrel{\circ}{\mathscr{M}}_{1} \text {. }
$$

Solution of this equation in block form is

$$
\left[S_{1}\right]_{m n}=-\frac{1}{\xi_{m}-\xi_{n}}\left[\stackrel{\circ}{\mathscr{M}}_{1}\right]_{m n}, \quad m \neq n, \quad m, n=1,2, \ldots,
$$

while diagonal blocks of $S_{1}$ are zeros.

In a similar manner one can find first two terms of the expansion (6.2)

$$
\begin{aligned}
& \mathscr{X}_{1}=\operatorname{diag} \mathscr{M}_{1}=\overline{\mathscr{M}}_{1}, \\
& \mathscr{X}_{2}=\frac{1}{2} \operatorname{diag}\left[\stackrel{\circ}{\mathscr{M}}_{1}, S_{1}\right] .
\end{aligned}
$$

Implementing the above procedure we obtain expansion of the first block $X^{(1)}$ of matrix $\mathscr{X}=\operatorname{diag}\left\{X^{(1)}, X^{(2)}, \ldots\right\}$ corresponding to the eigenvalue $\xi=2 \pi^{2}$ of multiplicity three:

$$
X^{(1)}=2 \pi^{2} I+2 \eta\left(\begin{array}{c}
q\left(k_{1}, k_{2}, k_{3}\right), 0,0 \\
0, q\left(k_{2}, k_{3}, k_{1}\right), 0 \\
0,0, q\left(k_{3}, k_{1}, k_{2}\right)
\end{array}\right)+\eta^{2} X_{2}^{(1)}+O\left(\eta^{3}\right),
$$

where $I$ is the $3 \times 3$ identity matrix (hereafter its dimension will be clear from the content), $q(x, y, z)=-\cos x+\cos y+\cos z+3$, and $X_{2}^{(1)}$ is the first block of the matrix $\mathscr{X}_{2}(6.11)$.

The second perturbed eigenvalue is defined from the matrix

$$
\begin{aligned}
X^{(2)}= & 3 \pi^{2} I+2 \eta\left(\begin{array}{cc}
2 \cos k_{1}+\cos k_{2}+\cos k_{3}+4, & -\frac{\sqrt{3}}{3} \cos k_{2}+\frac{\sqrt{3}}{3} \cos k_{3} \\
-\frac{\sqrt{3}}{3} \cos k_{2}+\frac{\sqrt{3}}{3} \cos k_{3}, \frac{2}{3} \cos k_{1}+\frac{5}{3} \cos k_{2}+\frac{5}{3} \cos k_{3}+4
\end{array}\right) \\
& +\eta^{2} X_{2}^{(2)}+O\left(\eta^{3}\right),
\end{aligned}
$$

where $X_{2}^{(2)}$ is the second block of the matrix $\mathscr{X}_{2}(6.11)$.

For the third perturbed eigenvalue we obtain the system

$$
X^{(3)}=5 \pi^{2} I+2 \eta\left(\begin{array}{c}
t\left(k_{1}, k_{2}, k_{3}\right), 0,0,0,0,0 \\
0, t\left(k_{1}, k_{3}, k_{2}\right), 0,0,0,0 \\
0,0, t\left(k_{2}, k_{1}, k_{3}\right), 0,0,0 \\
0,0,0, t\left(k_{3}, k_{1}, k_{2}\right), 0,0 \\
0,0,0,0, t\left(k_{2}, k_{3}, k_{1}\right), 0 \\
0,0,0,0,0, t\left(k_{3}, k_{2}, k_{1}\right)
\end{array}\right)+\eta^{2} X_{2}^{(3)}+O\left(\eta^{3}\right),
$$


TABLE 6.1

Quadratic approximation of the first 11 upper bounds of the spectral bands.

\begin{tabular}{|c|c|c|c|}
\hline $\begin{array}{l}\text { Eigenvalue } \\
\xi \xi\end{array}$ & Multiplicity & Indices $\check{p}$ & $\begin{array}{l}\text { Upper bound } \\
\text { approximation }\end{array}$ \\
\hline $2 \pi^{2}$ & 3 & $(0,1,1),(1,0,1),(1,1,0)$ & $2 \pi^{2}+12 \eta-2.01 \eta^{2}$ \\
\hline $3 \pi^{2}$ & 2 & $(1,1,1)_{ \pm}$ & $3 \pi^{2}+16 \eta-2.31 \eta^{2}$ \\
\hline $5 \pi^{2}$ & 6 & $\begin{array}{l}(0,1,2),(0,2,1),(1,0,2) \\
(2,0,1),(1,2,0),(2,1,0)\end{array}$ & $5 \pi^{2}+12 \eta-1.29 \eta^{2}$ \\
\hline $6 \pi^{2}$ & 6 & $(1,1,2)_{ \pm},(1,2,1)_{ \pm},(2,1,1)_{ \pm}$ & $6 \pi^{2}+16 \eta-1.43 \eta^{2}$ \\
\hline $8 \pi^{2}$ & 3 & $(0,2,2),(2,0,2),(2,2,0)$ & $8 \pi^{2}+12 \eta-1.08 \eta^{2}$ \\
\hline $9 \pi^{2}$ & 6 & $(1,2,2)_{ \pm},(2,1,2)_{ \pm},(2,2,1)_{ \pm}$ & $9 \pi^{2}+16 \eta-0.30 \eta^{2}$ \\
\hline $10 \pi^{2}$ & 6 & $\begin{array}{l}(0,1,3),(0,3,1),(1,0,3) \\
(3,0,1),(1,3,0),(3,1,0)\end{array}$ & $10 \pi^{2}+12 \eta-0.97 \eta^{2}$ \\
\hline $11 \pi^{2}$ & 6 & $(1,1,3)_{ \pm},(1,3,1)_{ \pm},(3,1,1)_{ \pm}$ & $11 \pi^{2}+12 \eta-1.28 \eta^{2}$ \\
\hline $12 \pi^{2}$ & 2 & $(2,2,2)_{ \pm}$ & $12 \pi^{2}+12 \eta-0.82 \eta^{2}$ \\
\hline $13 \pi^{2}$ & 6 & $\begin{array}{l}(0,2,3),(0,3,2),(2,0,3) \\
(3,0,2),(2,3,0),(3,2,0)\end{array}$ & $13 \pi^{2}+12 \eta-1.00 \eta^{2}$ \\
\hline $14 \pi^{2}$ & 12 & $\begin{array}{l}(1,2,3)_{ \pm},(1,3,2)_{ \pm},(2,3,1)_{ \pm} \\
(2,1,3)_{ \pm},(3,1,2)_{ \pm},(3,2,1)_{ \pm}\end{array}$ & $14 \pi^{2}+12 \eta-0.27 \eta^{2}$ \\
\hline
\end{tabular}

where $t(x, y, z)=-\cos x+\frac{2}{5} \cos y-\frac{8}{5} \cos z+3$. The calculations for the first 11 eigenvalues are given in Table 6.1.

Table 6.1 shows quadratic approximation of the upper bounds of the first 11 spectral bands. The linear term of the approximation is given exactly whose value is defined by the following rule. If among the indices of the eigenvalue $\xi$ there are indices of multiplicity two (which do not contain zeros), then the linearized upper bound of the band has the form $\xi+16 \eta$. Otherwise, when all indices of the eigenvalue $\xi$ have multiplicity one and contain zeros, its upper bound of the spectral band in the linear approximation is $\xi+12 \eta$.

7. Numerical results. Figure 7.1 shows dependence of the spectral bands of $\xi=\omega^{2} / c^{2}$ calculated numerically (shaded areas) on the perturbation parameter $\eta=$ $(\varepsilon \delta)^{-1}$. The eigenvalues were calculated through truncation of the matrix $\mathscr{M}$ to the order 2273 yielding 138 distinct eigenvalues of the greatest multiplicity 48 . The computations were carried out on the SGI Origin 2000 parallel computer on a uniform $10 \times 10 \times 10$ grid over the reduced Brillouin zone. Dotted lines indicate the upper bound of the bands. Dashed lines denote the upper bound of the bands in the linear approximation, while solid lines correspond to the quadratic approximation (see Table 6.1). The latter agrees closely with the numerical bound to the point of closing the gaps $(\eta \approx 2)$. 

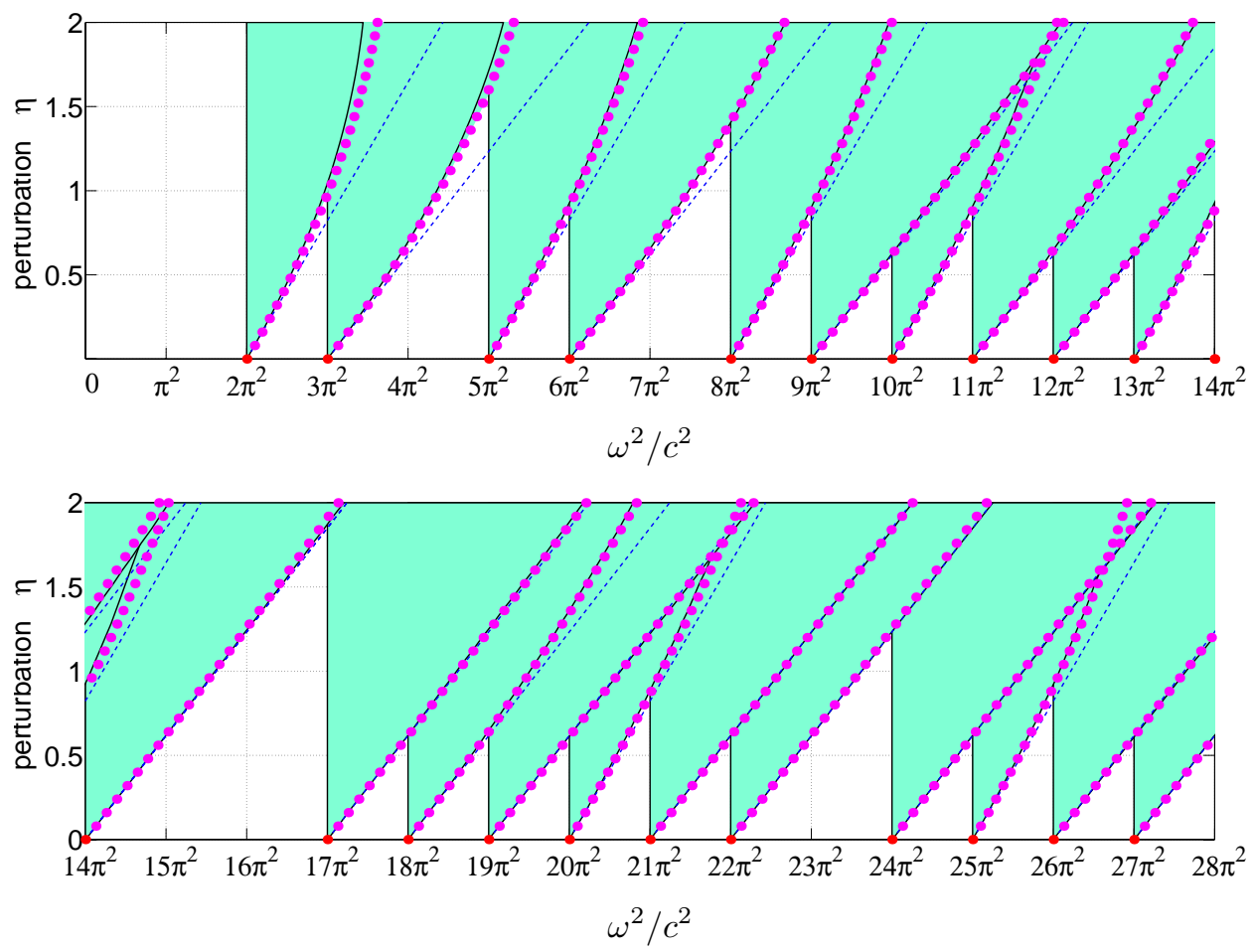

FIG. 7.1. Dependence of the spectral bands of photonic crystal calculated numerically (shaded areas enclosed by solid curves) on the perturbation parameter $\eta=(\varepsilon \delta)^{-1}$. Dashed lines denote the upper bound of the bands in linear approximation calculated explicitly. Dotted lines correspond to quadratic approximation.

Since spectral bands in the model depend only on the parameter $(\varepsilon \delta)^{-1}$, one can find the minimum value of the permittivity $\varepsilon_{\min }$ for which the first gap opens up

$$
\varepsilon_{\min }=\frac{1}{2\left[1-(1-f)^{1 / 3}\right]},
$$

where $f$ is the filling fraction of the optically dense dielectric. Figure 7.1 also suggests that for high frequencies the linear approximation gives good estimations of the spectral gaps.

\section{REFERENCES}

[1] W. Axmann, P. Kuchment, and L. Kunyansky, Asymptotic methods for thin high-contrast two-dimensional PBG materials, J. Lightwave Technology, 17 (1999), pp. 1996-2007.

[2] V. N. Bogaevski And A. Povzner, Algebraic Methods in Nonlinear Perturbation Theory, Springer-Verlag, New York, 1991.

[3] P. Braun And P. Wiltzius, Electrochemically grown photonic crystals, Nature, 402 (1999), pp. 603-604.

[4] G. Bouchitté, Analyse Limite de la Diffraction d'ondes Électromagnétiques par une Structure Mince, C. R. Acad. Sci. Paris Sér. II Méc. Phys. Chim. Sci. Univers. Sci. Terre, 311 (1990), pp. $51-56$.

[5] G. Bouchitté And R. Petit, On the concepts of a perfectly conducting material and of a perfectly conducting and infinitely thin screen, Radio Science, 24 (1989), pp. 13-26.

[6] M. Campbell, D. N. Sharp, M. T. Harrison, R. G. Denning, and A. J. Turberfield, 
Fabrication of photonic crystals for the visible spectrum by holographic lithography, Nature, 404 (2000), pp. 53-56.

[7] A. Chutinan AND S. NodA, Highly confined waveguides and waveguide bends in threedimensional photonic crystal, Appl. Phys. Lett., 75 (1999), pp. 3739-3741.

[8] A. Chutinan And S. NodA, Effects of structural fluctuations on the photonic bandgap during fabrication of a photonic crystal: A study of a photonic crystal with a finite number of periods, J. Opt. Soc. Amer. B Opt. Phys, 16 (1999), pp. 1398-1402.

[9] A. Chutinan and S. NodA, Spiral three-dimensional photonic band-gap structure, Phys. Rev. B, 57 (1998), pp. 2006-2008.

[10] C. T. Chan, W. Y. Zhang, Z. L. Wang, X.-Y. Lei, D. Zheng, W. Y. Tam, and P. Sheng, Photonic band gaps from metallo-dielectric spheres, Phys. B, 279 (2000), pp. 150-154.

[11] A. Figotin, High-contrast photonic crystals, in Diffusive Waves in Complex Media, J. P. Fouque, ed., Kluwer Academic Publishers, Dordrecht, The Netherlands, 1999, pp. 109136.

[12] A. Figotin And Yu. Godin, The computation of spectra of some 2D photonic crystals, J. Comput. Phys., 136 (1997), pp. 585-598.

[13] A. Figotin and P. Kuchment, Asymptotic Models of High-Contrast Periodic Dielectric and Acoustic Media, preprint.

[14] A. Figotin and P. Kuchment, Band-gap structure of the spectra of periodic dielectric and acoustic media. I. Scalar model, SIAM J. Appl. Math., 56 (1996), pp. 68-88.

[15] A. Figotin And P. Kuchment, Band-gap structure of the spectra of periodic dielectric and acoustic media. II. Two-dimensional photonic crystals, SIAM J. Appl. Math., 56 (1996), pp. 1561-1620.

[16] A. Figotin And P. Kuchment, Spectral properties of classical waves in high-contrast periodic media, SIAM J. Appl. Math., 58 (1998), pp. 683-702.

[17] Y. Fink, A. M. Urbas, M. G. Bawendi, J. D. Joannopoulos, and E. L. Thomas, Block copolymers as photonic bandgap materials, J. Lightwave Technology, 17 (1999), pp. 19631969.

[18] B. Goss Levi, Visible progress made in three-dimensional photonic crystals, Physics Today, January 1999, pp. 17-19.

[19] G. Guida, D. Maystre, G. Tayeb, and P. Vincent, Mean-field theory of two-dimensional metallic photonic crystals, J. Opt. Soc. Amer. B Opt. Phys., 15 (1998), pp. 2308-2315.

[20] G. Hardy and E. Wright, An Introduction to the Theory of Numbers, 5th ed., Clarendon Press, Oxford, 1996.

[21] S. John AND K. BusCh, Photonic bandgap formation and tunability in certain self-organizing systems, J. Lightwave Technology, 17 (1999), pp. 1931-1943.

[22] H. Kosaka, T. Kawashima, A. Tomita, M. Notomi, T. Tamamura, T. Sato, and S. KAWAKAMI, Superprism phenomena in photonic crystals: Toward microscale lightwave circuits, J. Lightwave Technology, 17 (1999), pp. 2032-2038.

[23] P. Kuchment, Floquet Theory for Partial Differential Operators, Birkhäuser-Verlag, Basel, 1993.

[24] P. Kuchment and L. Kunyansky, Spectral properties of high contrast band-gap materials and operators on graphs, Experiment. Math., 8 (1999), pp. 1-28.

[25] S. Lin And J. Fleming, A three-dimensional optical photonic crystal, J. Lightwave Technology, 17 (1999), pp. 1944-1947.

[26] R. C. McPhedran, N. A. Nicorovici, L. C. Botten, C. M. de Sterke, P. A. Robinson, And A. A. Asatryan, Anomalous absorpance by stacked metallic cylinders, Optics Comm., 168 (1999), pp. 47-53.

[27] K. Macintosh, O. McMahon, and S. Verghese, Three-dimensional metallodielectric photonic crystals incorporating flat metal elements, Microwave and Optical Technology Lett., 17 (1998), pp. 153-156.

[28] A. Moroz, Three-dimensional complete photonic band-gap structures in the visible, Phys. Rev. Lett., 83 (1999), pp. 5274-5277.

[29] A. Moroz And C. Sommers, Photonic band-gaps of three-dimensional face-centered cubic lattices, J. Phys. Condens. Matter, 11 (1999), pp. 997-1008.

[30] N. A. Nicorovici, R. C. McPhedran, and L. C. Botten, Photonic band gaps for arrays of perfectly conducting cylinders, Phys. Rev. E, 52 (1995), pp. 1135-1145.

[31] S. Noda, N. Yamamoto, M. Imada, H. Kobayashi, and M. Okano, Alignment and stacking of semiconductor photonic bandgaps by waver-fusion, J. Lightwave Technology, 17 (1999), pp. 1948-1955.

[32] J. B. Pendry, A. J. Holden, D. J. Robbins, and W. J. Stewart, Extremely low frequency plasmons in metallic mesostructures, Phys. Rev. Lett., 76 (1996), pp. 4773-4776. 
[33] R. Petit And M. Cadilhac, Electromagnetic theory of gratings: Some advances and some comments on the use of the operator formalism, J. Opt. Soc. Amer. A, 7 (1990), pp. $1666-1674$.

[34] R. Petit And G. TAYeB, Theoretical and numerical study of gratings consisting of periodic arrays of thin and lossy strips, J. Opt. Soc. Amer. A, 7 (1990), pp. 1686-1692.

[35] M. Reed And B. Simon, Analysis of Operators, Vol. 4, Academic Press, New York, 1978.

[36] T. J. Shepherd, P. J. Roberts, and R. Loudon, Soluble two-dimensional photonic-crystal model, Phys. Rev. E, 55 (1997), pp. 6024-6038.

[37] G. Subramanian, V. N. Manoharan, J. D. Thorne, and D. J Pine, Ordered macroporous materials by colloidal assembly: A possible route to photonic bandgap materials, Advanced Materials, 11 (1999), pp. 1261-1265.

[38] K. Sokoda AND J. KaWAmata, Novel approach to photonic bands with frequency-dependent dielectric constants, Optics Express, 3 (1998), pp. 12-18.

[39] S. Sujecki, P. Sewell, T. M. Benson, and P. C. Kendal, Novel beam propagation algorithms for tapered optical structures, J. Lightwave Technology, 17 (1999), pp. 2379-2388.

[40] H. B. Sun, J. F. Song, Y. Xu, S. Matsuo, H. Misawa, G. T. Du, and S. Y. Liu, Growth and properly characterizations of photonic crystal structures consisting of colloidal microparticles, J. Opt. Soc. Amer. B Opt. Phys., 17 (2000), pp. 476-480.

[41] R. TAO, Apply the electrorheological effect to produce three-dimensional photonic crystals for laser applications, Internat. J. Modern Phys. B, 13 (1999), pp. 2189-2196.

[42] M. S. Thijssen, R. Sprik, J. E. G. J. Wijnhoven, M. Megens, T. Narayanan, A. Lagendijk, AND W. L. VOs, Inhibited light propagation and broadband reflection in photonic air-sphere crystals, Phys. Rev. Lett., 83 (1999), pp. 2730-2733.

[43] O. Velev and E. Kaler, Structured porous materials via colloidal crystal templating: From inorganic oxides to metals, Advanced Materials, 12 (2000), pp. 531-534.

[44] Y. XIA, B. Gates, AND S. PARK, Fabrication of three-dimensional photonic crystals for use in the spectral region from ultraviolet to near-infrared, J. Lightwave Technology, 17 (1999), pp. 1956-1962.

[45] Z. Y. YuAn, J. W. Haus, AND K. SaKoda, Eigenmode symmetry for simple cubic lattices and the transmission spectra, Optics Express, 3 (1998), pp. 19-27.

[46] L. ZAVIeH AND T. MAYER, Demonstration of a three-dimensional simple-cubic infrared photonic crystal, Appl. Phys. Lett., 75 (1999), pp. 2533-2535. 